\title{
SU(3) Model Description of Be Isotopes
}

\author{
Eduardo de Almeida and S. Shelly Sharma \\ Departamento de Física, Universidade Estadual de Londrina, 86051-990, Londrina, PR, Brazil
}

Received on 20 October, 2003

\begin{abstract}
Experimental data on light nuclei close to dripline suggests that as the nucleon number asymmetry increases, the shell structure from stability line is not preserved. In contrast with spherical shell model, Elliott's SU(3) model, uses a deformed multi-nucleon basis to describe nuclear states. The SU(3) symmetry is a strong feature of $1 \mathrm{p}$ shell nuclei, where symmetry breaking spin-orbit force is rather weak. We have calculated the binding energies and low-lying energy spectra of Be isotopes $(A=6$ to $A=14)$, within the framework of Elliott's SU(3) model, with special emphasis on effects due to the presence of intruder orbit $1 \mathrm{~S}_{1 / 2}$ in the region. The model space includes $\mathrm{SU}(3)$ basis states that maximize the quadrupole-quadrupole interaction $(-\chi Q \cdot Q)$. An extended model space includes, in addition, a set of $2 \mathrm{p}-2 \mathrm{~h}$ excitations, with excited nucleons occupying lowest energy SU(3) states in harmonic oscillator shell $\mathrm{N}=2$. Group theoretical methods have been used to classify the states and calculate the nuclear interaction matrix elements. Good angular momentum states are projected out from intrinsic deformed $\mathrm{SU}(3)$ states by using standard angular momentum projection techniques. The interaction used contains the monopole-monopole, quadrupole-quadrupole and isospin dependent terms. Interaction parameters are fixed so as to reproduce the binding of 4 nucleons in $\mathrm{N}=1$ orbit for the $\mathrm{N}=\mathrm{Z}$ isotope- ${ }^{8} \mathrm{Be}$ in ground state and first excited $2^{+}$state, along with the known systematics of single neutron separation energies for Be isotopes. The calculated energy espectra are compared with available experimental data. The calculated excitation energies of intruder states in ${ }^{8} \mathrm{Be}$ and ${ }^{10} \mathrm{Be}$, are discussed in the light of available experimental evidence and theoretical results from shell model as well as deformed oscillator model calculations of Fayache et al..
\end{abstract}

\section{Introduction}

Shell model predictions for ground state spins and parities of Be isotopes with $6 \leq A \leq 10$ and $A=12,14$, are verified experimentally. For ${ }^{11} \mathrm{Be}$ and ${ }^{13} \mathrm{Be}$ nuclei, however, the model predicts ground state spin and parity values of $1 / 2^{-}$and $5 / 2^{+}$, to be compared with the experimental values of $1 / 2^{+}$and $1 / 2^{-}$[1], respectively. Low lying spectra of ${ }^{9} \mathrm{Be},{ }^{11} \mathrm{~N},{ }^{11} \mathrm{Be}$ and ${ }^{13} \mathrm{Be}$, also point to a change in nuclear structure close to the drip line. The presence of positive parity intruder orbit $1 \mathrm{~S}_{1 / 2}$, in the region of $\mathrm{N}=1$ single particle orbits, offers a possible explanation for experimentally observed spin and parity values. As a consequence, intruder states are expected to be found in low lying spectra of Be isotopes $[2,3,4]$.

The object of the present work is to understand the nuclear structure of $\mathrm{Be}$ isotopes in the low-energy region, within the framework of SU(3) model. The SU(3) model was proposed and used by Elliott [5] to describe rotational bands in light nuclei. Appropriate representations $(\lambda \mu)$ of symmetry group SU(3) are used, in this model, to generate multi-nucleon basis states for a given nucleus. Using a four parameter residual interaction with monopolemonopole, quadrupole-quadrupole and isospin dependent interaction terms with strength parameters $\left(P_{0}, P_{1}\right), \chi$, and $\beta$ respectively, we have calculated the binding energies and low-lying spectra of Be isotopes. The ground state band is described by the representation $(\lambda \mu)$ that maximizes the ground state binding energy for a given strength $\chi$ of quadrupole-quadrupole interaction. An extended model space including $(2 \mathrm{p}-2 \mathrm{~h})$ excitations is then used to obtain the likely position of intruder state in ${ }^{8,10,12} \mathrm{Be}$. Interaction parameters $\chi$ and $P_{0}$ are fitted to experimental ground state binding energy and excitation energy of $2_{1}^{+}$state in $\mathrm{N}=\mathrm{Z}$ isotope ${ }^{8} \mathrm{Be}$. Interaction parameters $P_{1}$ and $\beta$ are adjusted to give a best fit to experimental ground state binding energies of Be isotopes with $6 \leq A \leq 14$. The calculated energy spectra are compared with available experimental data. The New Mass Formula (NMF) of reference [6], that made possible the identification and prediction of new magic numbers, is also used to calculate the residual interaction. A comparison of results from NMF, our calculation and experimental data shows that the interaction used by us reproduces reasonably well the experimental ground state binding energies and neutron separation energies for Be isotopes.

\section{SU(3) Model}

Symmetry group SU(3) [7] has found many applications in nuclear physics as well as particle physics. We outline the main features of SU(3) model of Elliott [5], writing down the generators, subgroups and relevant Casimir operators. An SU(3) representation is characterized by quantum numbers $(\lambda \mu)$, that determine the eigenvalue of Casimir operator for symmetry group $\mathrm{SU}(3)$. The states belonging to a given representation can be further labeled by quantum numbers that determine the eigenvalues of Casimir operators for subgroups of SU(3). Multi-nucleon deformed states, having good orbital angular momentum, can be projected out from a state belonging to a given representation of SU(3) group by using angular momentum projection techniques. 
TABLE I. Casimir operators, Casimir operator eigenvalues and quantum numbers of SU(3) group and subgroups.

\begin{tabular}{|c|c|c|c|c|}
\hline & $\mathrm{SU}(3)$ & $\mathrm{SU}(2)$ & $\mathrm{U}_{1}(1)$ & $\mathrm{U}_{2}(1)$ \\
\hline Casimir op. & $\frac{(Q . Q+3 L . L)}{6}$ & $\frac{\left(3 L_{0}^{2}+Q_{2} Q_{-2}+Q_{-2} Q_{2}\right)}{12}$ & $L_{0} / 2$ & $Q_{0}$ \\
\hline eigenvalue & $\frac{2}{3}\left(\lambda^{2}+\mu^{2}+\lambda \mu+3 \lambda+3 \mu\right)$ & $\Lambda(\Lambda+1)$ & $K / 2$ & $\epsilon$ \\
\hline quantum number & $(\lambda \mu)$ & $\Lambda$ & $K$ & $\epsilon$ \\
\hline
\end{tabular}

In spherical basis, three components of orbital angular momentum operator $L$, and five components of quadrupole moment operator $Q$ constitute the eight generators of symmetry group $\mathrm{SU}(3)$. A component of quadrupole moment, is defined as

$Q_{q}=\sqrt{\frac{4 \pi}{5}} \alpha^{2}\left(r^{2} Y_{2, q}(\widehat{r})+\frac{p^{2}}{\alpha^{2} \hbar} Y_{2, q}(\widehat{p})\right), \quad q=0, \pm 1, \pm 2$

where $\alpha^{2}=m \omega / \hbar$ and $Y_{2, q}$ are the spherical harmonics. The eight SU(3) generators form a Lie algebra and satisfy the following commutation relations,

$$
\begin{aligned}
{\left[L_{q}, L_{q^{\prime}}\right] } & =-\sqrt{2}\left(11 q q^{\prime} \mid 1 q+q^{\prime}\right) L_{q+q^{\prime}}, \\
{\left[Q_{q}, L_{q^{\prime}}\right] } & =-\sqrt{6}\left(21 q q^{\prime} \mid 2 q+q^{\prime}\right) Q_{q+q^{\prime}}, \\
{\left[Q_{q}, Q_{q^{\prime}}\right] } & =3 \sqrt{10}\left(22 q q^{\prime} \mid 1 q+q^{\prime}\right) L_{q+q^{\prime}} .
\end{aligned}
$$

A convenient labeling scheme can be achieved by noting that the generators of symmetry group $\mathrm{SU}(2)$ are proportional to $Q_{2}, Q_{-2}$ and $L_{0}$. Next $L_{0}$ and $Q_{0}$ are identified as the generators of subgroups $U_{1}(1)$ and $U_{2}(1)$, respectively. We show in Table I the relevant Casimir operators, Casimir operator eigenvalues, and the corresponding quantum numbers for symmetry group SU(3) and it's subgroups. The quantum numbers $(\lambda \mu)$ characterize the permutation symmetry between harmonic oscillator quanta and $\Lambda$ is proportional to the number of quanta in $x y$ plane for the case $\mu=0[8,9]$.

Elliott [5] has shown that we can expand a state $\chi\left((\lambda \mu) \epsilon_{\max } \Lambda K\right)$ belonging to $\mathrm{SU}(3)$ representation $(\lambda \mu)$ in terms of states $\Psi((\lambda \mu) K L K)$, having good angular momentum quantum number $L$ that is

$$
\chi\left((\lambda \mu) \epsilon_{\max } \Lambda K\right)=\sum_{L} c(\lambda \mu K, L) \Psi((\lambda \mu) K L K),
$$

where the coefficients $c$ are real and $\epsilon_{\max }$ is the highest eigenvalue of operator $Q_{0}$. We use the method of angular momentum projection and obtain the wave functions $\Psi((\lambda \mu) K L K)$, which are in turn used to obtain analytically the matrix elements of operator Q.Q. A fortran routine has been written to calculate $c$ 's and eventually matrix elements of $Q . Q$ operator between relevant good $L$ states.

\section{Interaction Parameters}

The nuclei studied are the isotopes of Be with $6 \leq A \leq 14$. We define the binding energy of isotope $\mathrm{A}$ in residual interaction zero state, $I(\mathrm{~A})$ as

$I(A)=B(A, Z)-B(4,2)-2 S_{p}(5,3)-(A-6) S_{n}(5,2)$, where $B(\mathrm{~A}, Z)$ is the binding energy of the nucleus with $Z$ protons and A-Z neutrons, $S_{p}(5,3)$ is one proton separation energy of ${ }^{5} \mathrm{Li}$ and $S_{n}(5,2)$ is one neutron separation energy of ${ }^{5} \mathrm{He}$. All terms on the right hand side in Eq. 1 can be obtained from available experimental data [1]. The experimental ground state residual interaction between active nucleons, $I(\mathrm{~A})$, serves as a constraint on interaction parameters used in theoretical calculations.

We use an interaction Hamiltonian containing monopole-monopole, quadrupole-quadrupole and isospin dependent interaction terms that is

$$
H_{\text {res }}=-F_{0}(\underline{n})-\chi Q \cdot Q+\beta T(T+1),
$$

where $\underline{n}$ is the number of active nucleons $(\underline{n}=\mathrm{A}-4)$. The isospin dependent interaction is repulsive while the monopole-monopole and quadrupole-quadrupole interactions are attractive. The strength of monopole-monopole interaction for nucleons in the same shell is $P_{0}$ and for nucleons in different shells is $P_{1}$. We write the monopolemonopole interaction for $n_{1}$ and $n_{2}\left(\underline{n}=n_{1}+n_{2}\right)$ nucleons in oscillator shells $\mathrm{N}=1$ and $\mathrm{N}=2$, respectively, as

$$
F_{0}(\underline{n})=P_{0} \frac{n_{1}\left(n_{1}-1\right)}{2}+P_{0} \frac{n_{2}\left(n_{2}-1\right)}{2}+P_{1} n_{1} n_{2} .
$$

From Table I, the quadrupole-quadrupole interaction operator may be expressed as

$$
-\chi Q \cdot Q=-\chi 3\left(2 C_{S U(3)}-L . L\right),
$$

where $C_{S U(3)}$ is the Casimir operator of the SU(3) group. The ground state isospin is defined as $T=\left|\frac{(n-p)}{2}\right|$, where $n$ and $p$ are the number of neutrons and protons respectively.

\subsection{New Mass Formula (NMF)}

Comparing the mass formula of Bethe-Weizsacker [10, 11] with the New Mass Formula (NMF) [6], the NMF has a new parameter $\Delta(\mathrm{N}, \mathrm{Z})$ and has a redefinition of the pairing term $\delta_{\text {new }}$. The NMF is written as

$$
\begin{gathered}
B(A, Z)_{n e w}=a_{v} A-a_{s} A^{2 / 3}-a_{c} \frac{Z(Z-1)}{A^{1 / 3}} \\
-a_{\text {sym }} \frac{(A-2 Z)^{2}}{A}+\delta_{\text {new }}+\Delta(N, Z),
\end{gathered}
$$


TABLE II. Values of $\left(\lambda_{1} \mu_{1} L_{1}\right)$ and $\left(\lambda_{2} \mu_{2} L_{2}\right)$ for active nucleons in $\mathrm{N}=1$ and $\mathrm{N}=2$ oscillator shells.

\begin{tabular}{|c|c|c|c|c|c|c|c|c|}
\hline \multicolumn{5}{|c|}{ g. s. band } & \multicolumn{5}{c|}{ 2p-2h excitations } \\
\hline & $\lambda_{1} \mu_{1}$ & $L_{1}$ & $\lambda_{2} \mu_{2}$ & $L_{2}$ & $\lambda_{1} \mu_{1}$ & $L_{1}$ & $\lambda_{2} \mu_{2}$ & $L_{2}$ \\
\hline${ }^{6} B e$ & 20 & 0,2 & & & & & & \\
\hline${ }^{7} B e$ & 30 & 1,3 & & & & & & \\
\hline${ }^{8} B e$ & 40 & $0,2,4$ & & & 20 & 0,2 & 40 & $0,2,4$ \\
\hline${ }^{9} B e$ & 31 & $1,2,3,4$ & & & 30 & 1,3 & 40 & $0,2,4$ \\
\hline${ }^{10} B e$ & 22 & $0,2^{2}, 3,4$ & & & 40 & $0,2,4$ & 40 & $0,2,4$ \\
\hline${ }^{11} B e$ & 22 & $0,2^{2}, 3,4$ & 20 & 0,2 & 40 & $0,2,4$ & 41 & $1,2,3,4,5$ \\
\hline${ }^{12} B e$ & 22 & $0,2^{2}, 3,4$ & 40 & $0,2,4$ & 20 & 0,2 & & \\
\hline${ }^{13} B e$ & 21 & $1,2,3$ & 40 & $0,2,4$ & 31 & $1,2,3,4$ & 42 & $0,2^{2}, 4^{2}, 3,5,6$ \\
\hline${ }^{14} B e$ & 20 & 0,2 & 40 & $0,2,4$ & 22 & $0,2^{2}, 3,4$ & 42 & $0,2^{2}, 4^{2}, 3,5,6$ \\
\hline
\end{tabular}

where $a_{v}=15,85 \mathrm{MeV}, a_{s}=18,34 \mathrm{MeV}, a_{c}=0,71$ $\mathrm{MeV}, a_{\text {sym }}=23,21 \mathrm{MeV}, \delta_{\text {new }}=\left(1-\exp \left(-\frac{Z}{c}\right)\right) \delta$, $c=6 / \operatorname{Ln} 2, \delta$ is equal to $12 A^{-1 / 2}$ for even-even nuclei, $-12 A^{-1 / 2}$ for odd-odd nuclei, 0 for odd A nuclei and $\Delta(N, Z)=\left|N-\frac{4}{3} Z\right| N^{k} Z \exp \left(-\frac{Z}{3}\right)$ with $k=0,45$.

Using the NMF to calculate the binding energy we obtain the residual interaction $I_{n e w}(\mathrm{~A})$ for Be isotopes as

$I_{\text {new }}(A)=B_{\text {new }}(A, 4)-B(4,2)-2 S_{p}(5,3)-(A-6) S_{n}(5,2)$.

\section{Model Space}

Table II lists the representations $(\lambda \mu)$ that maximize the quadrupole-quadrupole interaction, and the possible $L$ values for the ground state band. The representations $(\lambda \mu)$ and possible angular momenta for the case when two nucleons are excited out of ground state $(2 \mathrm{p}-2 \mathrm{~h}$ excitations) are also presented in Table II.

We predict correct spin and parity for ${ }^{11} \mathrm{Be}$ ground state if we consider the last neutron occupying $\mathrm{N}=2$ oscillator orbit. The isotopes, ${ }^{11} \mathrm{Be},{ }^{12} \mathrm{Be},{ }^{13} \mathrm{Be}$ and ${ }^{14} \mathrm{Be}$ have at least one neutron occupying the $\mathrm{N}=2$ oscillator shell, in the ground state. Therefore we must subtract $\hbar \omega$ from residual interaction for ${ }^{11} \mathrm{Be}$ and $2 \hbar \omega$ for ${ }^{12} \mathrm{Be},{ }^{13} \mathrm{Be}$ and ${ }^{14} \mathrm{Be}$. We use the formula $\hbar \omega=45 A^{-1 / 3}-40 A^{-2 / 3}$ to get the value of oscillator constant. In comparison Fayache et al [4] have used $\hbar \omega=45 A^{-1 / 3}-25 A^{-2 / 3}$, with a milder surface dependence.

\section{Results and Conclusions}

In Fig. 1 we plot the ground state binding energies of Be isotopes, due to residual interaction between active nucleons, (i) calculated by using SU(3) model, (ii) calculated from New Mass Formula (NMF Eq. 3) and (iii) obtained from experimental data (Eq. 1). The parameter $\chi=0.17 \mathrm{MeV}$ reproduces the excitation energy of $2_{1}^{+}$state in $\mathrm{N}=\mathrm{Z}$ isotope ${ }^{8} \mathrm{Be}$. For this choice of $\chi$, a parameter value of $P_{0}=2.3$ $\mathrm{MeV}$ is needed to obtain experimental ground state binding energy of ${ }^{8} \mathrm{Be}$. Interaction parameters $P_{1}$ and $\beta$ are next adjusted to give a best fit to experimental ground state binding energies of Be isotopes with $6 \leq A \leq 14$. The fit shown in Fig. 1 is obtained with $P_{1}=2.2 \mathrm{MeV}$ and $\beta=4.1 \mathrm{MeV}$. We can verify that the calculated binding energies for Be isotopes show a good agreement with experimental values. Correct ground state spins and parities are reproduced including the inversion of spectra in ${ }^{11} \mathrm{Be}$. We notice that the results of New Mass Formula, also reproduce the trend of experimental binding energies of nuclei obtained by adding neutrons to ${ }^{8} \mathrm{Be}$.

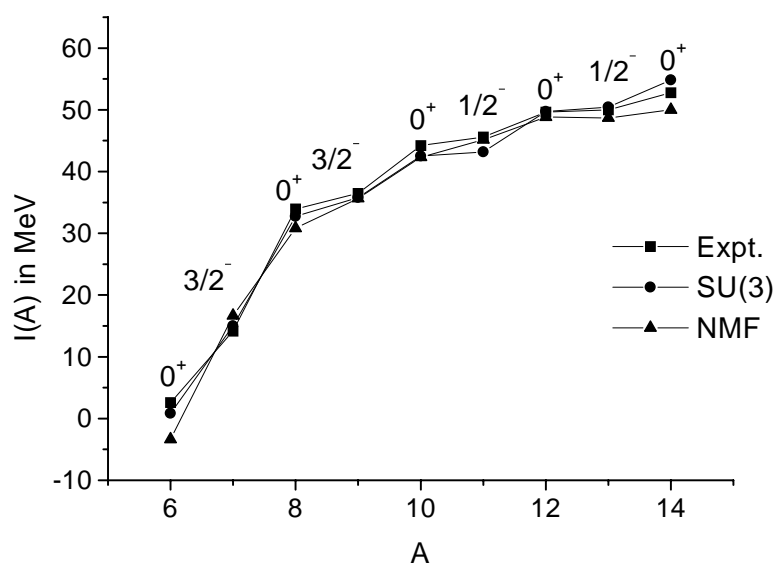

Figure 1. Experimental and calculated espectra for ${ }^{8} \mathrm{Be},{ }^{10} \mathrm{Be}$ and ${ }^{12} \mathrm{Be}$. Calculated $(2 \mathrm{p}-2 \mathrm{~h})$ excitation states are shown in square brackets.

In Fig. 2, we compare with experimental and calculated spectra for ${ }^{8,10,12} \mathrm{Be}$ obtained by using the interaction of Eq. 2 with the set of parameter values chosen earlier. In this calculation we have used the states defined by SU(3) representations and allowed angular momenta listed in Table II. The states obtained by using representations with $(2 \mathrm{p}-2 \mathrm{~h})$ excitations are shown in square brackets in Fig. 2. We observe that the low lying states of ${ }^{8} \mathrm{Be}$ are not likely to mix strongly with states from ( $2 p-2 h)$ excitations. Table III lists the calculated and experimental excitation energies of $0_{1}^{+}, 2_{1}^{+}, 0_{2}^{+}$, and $2_{2}^{+}$ states for comparison. The energy difference between the 
TABLE III. Excitation energies of $0_{1}^{+}, 2_{1}^{+}, 0_{2}^{+}$, and $2_{2}^{+}$states in ${ }^{8} \mathrm{Be},{ }^{10} \mathrm{Be}$ and ${ }^{12} \mathrm{Be}$ nuclei in $\mathrm{MeV}$. Square brackets represent states arising from $(2 \mathrm{p}-2 \mathrm{~h})$ excited configurations.

\begin{tabular}{|c|c|c|c|c|c|c|c|c|c|c|c|}
\hline \multicolumn{4}{|l|}{${ }^{8} \mathrm{Be}$} & \multicolumn{4}{|l|}{${ }^{10} \mathrm{Be}$} & \multicolumn{4}{|l|}{${ }^{12} \mathrm{Be}$} \\
\hline \multicolumn{2}{|l|}{ Calc. } & \multicolumn{2}{|c|}{ Expt. } & \multicolumn{2}{|l|}{ Calc. } & \multicolumn{2}{|c|}{ Expt. } & \multicolumn{2}{|l|}{ Calc. } & \multicolumn{2}{|c|}{ Expt. } \\
\hline $0_{1}^{+}$ & 0.0 & $0_{1}^{+}$ & 0.0 & $0_{1}^{+}$ & 0.0 & $0_{1}^{+}$ & 0.0 & $0_{1}^{+}$ & 0.0 & $0_{1}^{+}$ & 0.0 \\
\hline $2_{1}^{+}$ & 3.04 & $2_{1}^{+}$ & 3.04 & $2_{1}^{+}$ & 3.04 & $2_{1}^{+}$ & 3.37 & $2_{1}^{+}$ & 3.04 & $2_{1}^{+}$ & 2.10 \\
\hline$\left[0_{2}^{+}\right]$ & 18.68 & $0_{2}^{+}$ & 20.2 & {$\left[0_{2}^{+}\right]$} & 3.83 & $0_{2}^{+}$ & 6.18 & {$\left[0_{2}^{+}\right]$} & 2.98 & & \\
\hline$\left[2_{2}^{+}\right]$ & 21.70 & & & {$\left[2_{2}^{+}\right]$} & 6.86 & & & {$\left[2_{2}^{+}\right]$} & 6.01 & & \\
\hline
\end{tabular}

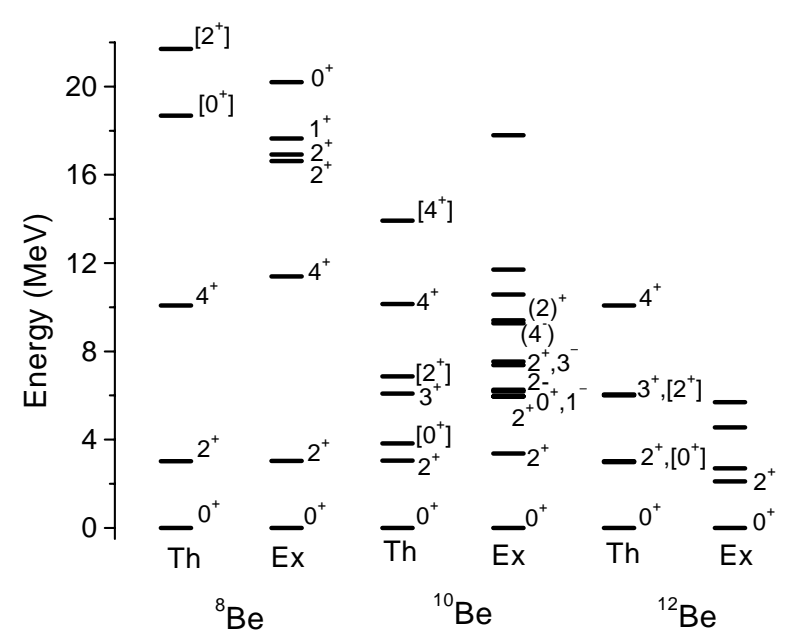

Figure 2. Calculated (SU(3) model and NMF) and experimental ground state binding energy due to residual interaction between active nucleons for Be Isotopes.

states $2_{2}^{+}$and $2_{1}^{+}(2 \mathrm{p}-2 \mathrm{~h})$ for ${ }^{8} \mathrm{Be}$ is $18.66 \mathrm{MeV}$ while for ${ }^{10} \mathrm{Be}$ and ${ }^{12} \mathrm{Be}$ it is just $3.82 \mathrm{MeV}$ and $3.97 \mathrm{MeV}$ respectively. Therefore the ground states as well as the $2_{1}^{+}$states in nuclei ${ }^{10} \mathrm{Be}$ and ${ }^{12} \mathrm{Be}$ are expected to have strong contributions from $(2 \mathrm{p}-2 \mathrm{~h})$ configurations. We are studying the effect of such mixing on radii and transitions of Be isotopes.

The calculated excitation energy of $0^{+}(2 \mathrm{p}-2 \mathrm{~h})$ intruder state in ${ }^{8} \mathrm{Be}$ is $18.6 \mathrm{MeV}$. Experimentally a $0^{+}$state is seen at $20.2 \mathrm{MeV}$. Using a deformed oscillator model calculation Fayache et al [4] have reported, a value of $17.23 \mathrm{MeV}$ in ${ }^{8} \mathrm{Be}$. As such our SU(3) model result is close to the calculated value from deformed oscillator model and not far from the experimentally observed $0^{+}$state energy. For the case of ${ }^{10} \mathrm{Be}$ our result of $3.83 \mathrm{MeV}$ and the value of $6.36 \mathrm{MeV}$ calculated in ref. [4] may possibly be compared with the energy of a $0^{+}$state seen at $6.18 \mathrm{MeV}$, experimentally. We may point out, however that our results are preliminary results. As pointed out by Fayache at el.[4], it is essential to take into consideration triaxial nature of the ground state in ${ }^{10} \mathrm{Be}$.

\section{Acknowledgements}

Financial support from CAPES, Brazil and Fundação Araucaria is acknowledged.

\section{References}

[1] National Nuclear Data Center, http://www.nndc.bnl.gov/nndc/ensdf/ .

[2] F. C. Barker, Aust. J. Phys. 41, 743 (1988).

[3] F. C. Barker, Aust. J. Phys. 42, 25 (1989).

[4] M. S. Fayache, E. Moya de Guerra, P. Sarriguren, Y. Y. Sharon, and L. Zamick, Phys. Rev. C 57, 2351 (1998).

[5] J. P. Elliott, Proc. R. Soc. (London) A 245, 128 (1958); Proc. R. Soc. (London) A 245, 562 (1958).

[6] C. Samanta and S. Adhikari, Phys. Rev. C 65, 037301 (2002).

[7] M. Hammermesh, Group Theory and its applications to Physical problems, Addison-Wesley, Reading MA 1962.

[8] V. G. Gueorguiev and J. P. Draayer, Revista Mexicana de Fisica 44, 2 (1999).

[9] A. Bohr and B. R. Mottelson, Nuclear Structure, V. 1, W. A. Benjamin, Inc., (1969).

[10] C. F. von Weizsäcker, Z. Physik 96, 431 (1935).

[11] H. Bethe, Rev. Mod. Phys. 8, 82 (1936). 